Research Article

\title{
Drug utilization pattern of antimicrobial drugs in intensive care unit of a tertiary care hospital attached with a medical college
}

\author{
Rutvij Hedamba*, Chintan Doshi, Nishita H. Darji, Bansari Patel, Varsha Kumari, \\ Hiren R. Trivedi
}

Department of Pharmacology, M P Shah Government Medical College, Jamnagar, Gujarat, India

Received: 6 December 2015

Accepted: 7 January 2016

*Correspondence to:

Dr. Rutvij H. Hedamba,

Email: rutvij237@yahoo.co.in

Copyright: (C) the author(s), publisher and licensee Medip Academy. This is an open-access article distributed under the terms of the Creative Commons Attribution Non-Commercial License, which permits unrestricted noncommercial use, distribution, and reproduction in any medium, provided the original work is properly cited.

\begin{abstract}
Background: A number of antimicrobial drugs are prescribed to the patients in Intensive Care Unit (ICU). Drug utilization research was defined by WHO as the study of marketing, distribution, prescription, and use of drugs in a society, with special emphasis on the resulting medical, social and economic consequences. There is lack of information about the use of antimicrobial agents in the ICU at GGGH till date. So we decided to conduct this type of study.

Methods: A prospective observational study was carried out for 12 months. Patients of age $>18$ of both sexes who was admitted in ICU were included in the study while pregnant and lactating women excluded. The prescribing pattern was analyzed by using World Health Organization basic drug indicators.

Results: Among 611 patients, 373 (61.04\%) were male and 238 (38.95\%) were female. The most common emergency was post-operative 204 $(33.38 \%)$.The most common prescribed antimicrobial drug group and drug was antiamoebic drugs and metronidazole respectively in 437 (24.04\%) out of total drugs prescribed. The numbers of antimicrobial drugs prescribed per patient were 2.97. 68\% drugs were prescribed from WHO and national EML list. Total $57.26 \%$ drugs were prescribed by generic name. Average antimicrobial drug cost per patient was 1805.34 rs.

Conclusions: Despite of limitations of present study it gives important conclusion about how antimicrobial drugs are used in GGGH ICU. This information can be used for improvement of current treatment strategies.
\end{abstract}

Keywords: Drug utilization, Antimicrobial drugs, Prescribing pattern, Cost analysis

\section{INTRODUCTION}

Drug utilization study has been defined by the World Health Organization (WHO) as "The marketing, distribution, prescription and uses of drugs in a society with special emphasis on the resulting medical and social and economical consequences." The interests in drug utilization studies were generated as a result of:

(1) Number of new drug marketed (2) Wide variations in the prescribing pattern (3) Growing concern about delayed adverse effects (4) Increasing concern about the cost of drug as reflected by increase in both sales and the volume of prescription drugs. ${ }^{2}$

Infectious diseases were the major cause of morbidity and mortality before discovery of antimicrobial agents. After accidental discovery of penicillin, there was decrease in morbidity and mortality. During the period of 1940-2000, many new antimicrobial agents like streptomycin, chloramphenicol, cephalosporins, fluroquinolones, extended spectrum penicillin as well as other antimicrobial agents are discovered. ${ }^{3}$

A number of drugs are prescribed to the patients during their hospitalization in Intensive Care Unit (ICU). Antimicrobial agents are prescribed for treatment of critical illness and for prevention of infections in critically ill patients who are having low immunity, increased susceptibility to virulent micro-organisms as well as during different medical procedures (catheterization) or use of medical devices (ventilator). ${ }^{4-6}$ 
Antimicrobial agents are widely prescribed for treatment and prevention of postoperative infections in surgical patients admitted in ICU. $^{7}$ In 2004, World Health Organisation (WHO) reported that $50 \%$ antimicrobial agents are used inappropriately all over the world. ${ }^{8}$ Inappropriate use of antimicrobial agents include wrong choice of antimicrobial for empirical as well as treatment against microorganisms, use in case wrong indications, incorrect dosage or duration of treatment. ${ }^{9}$

Present study was undertaken to analyse the pattern of drug utilization of antimicrobial medications in outdoor patients of ICU of a tertiary care teaching hospital in Jamnagar.

\section{METHODS}

An observational, prospective antimicrobial drug utilization study was carried out in ICU of Guru Gobind Singh Government Hospital, a tertiary care teaching hospital attached to Shri M. P. Shah Government Medical College, Jamnagar. Prior permission of the medical superintendent of G. G. G Hospital, institutional ethical committee and head of medicine department was obtained for conducting the study. My study duration was 1 year from July 2013 to June 2014.

\section{Development of the protocol}

An appropriate study protocol and pro-forma were developed and discussed with teaching staff members of the pharmacology department and head of department.

\section{Selection criteria of patient}

\section{Inclusion criteria:}

1) Adult patients above 18 years of age and of either gender who were admitted in medical, or surgical, Intensive Care Units (ICU) GGGH Hospital, Jamnagar.

2) Those patients or their relatives who gave consent and were willing to participate in this study.

\section{Exclusion criteria:}

1) Lactating and pregnant women. Collection of data.

Total 611 cases were analysed. The WHO drug indicators that were selected to analyze the prescribing pattern included: (1) Average number of the antimicrobial drugs prescribed per encounter. (2) Percentage of the antimicrobial drugs prescribed by generic name. (3) Percentage of the antimicrobial drugs prescribed from essential drug list. (4) Frequency of antimicrobial drugs usage as per patient. (5) Average antimicrobial drug cost per patient. (6) Most common cause for admission in ICU.

\section{RESULTS}

\section{Characteristics of study participants}

Out of total 611 patients percentage of female and male patients was $61.04 \%$ and $38.95 \%$ respectively. The relative distribution of different antimicrobial drugs in different age groups and genders is shown in (Table 1).

Table 1: Age \& sex distribution.

\begin{tabular}{|llll|}
\hline Age(year) & Male & Female & Total (\%) \\
\hline $18-37$ & $131(35.12 \%)$ & $99(41.59 \%)$ & $230(37.64 \%)$ \\
\hline $38-57$ & $127(34.08 \%)$ & $65(27.31 \%)$ & $192(31.42 \%)$ \\
\hline $58-77$ & $100(26.81 \%)$ & $60(25.21 \%)$ & $160(26.19 \%)$ \\
\hline $78-97$ & $15(4.0250 \%)$ & $14(5.88 \%)$ & $29(4.74 \%)$ \\
\hline Total & $373(61.04 \%)$ & $238(38.95 \%)$ & $611(100 \%)$ \\
\hline
\end{tabular}

\section{Pattern of diagnosis of patients admitted in ICU}

Majority of the patients were suffering from postoperative complication 204 (33.38\%). Other common conditions were Organophophorus poisoning 164 $(26.84 \%)$, accidental injuries $(7.20 \%)$ cerebral malaria $(5.56 \%)$ and others $27.02 \%$ (Table 2).

Table 2: Diagnosis of patients admitted in ICU.

\begin{tabular}{|c|c|c|c|c|}
\hline Disease & $\begin{array}{l}\text { Post- } \\
\text { operative } \\
\text { complicat } \\
\text { ion }\end{array}$ & $\begin{array}{l}\text { Organopho } \\
\text { phorus } \\
\text { poisoning }\end{array}$ & $\begin{array}{l}\text { Accide } \\
\text { ntal } \\
\text { injuries }\end{array}$ & $\begin{array}{l}\text { Cerebral } \\
\text { malaria }\end{array}$ \\
\hline $\begin{array}{l}\text { No. of } \\
\text { patients } \\
(\%)\end{array}$ & $\begin{array}{l}204 \\
(33.38 \%\end{array}$ & $\begin{array}{l}164 \\
(26.84 \%)\end{array}$ & $\begin{array}{l}44 \\
(7.20 \%)\end{array}$ & $\begin{array}{l}34 \\
(5.56 \%)\end{array}$ \\
\hline
\end{tabular}

Analysis of prescription patterns according to various WHO drug use indicators.

- The average number of drugs per encounter was 2.97 in our study.

- $68 \%$ drugs were prescribed from WHO essential drug list (2013) and National essential drug list (2011).

- $57.26 \%$ drugs were prescribed by generic name (Figure 3).

- Most commonly prescribed drug was metronidazole $437(24.04 \%)$ followed by ceftriaxone $316(17.23 \%)$ out of total 1816 drug prescribed to 611 patients (Figure 1).

- Most common drug group from which drugs prescribed was antiamoebic drug group 437 $(24.04 \%)$ followed by penicillin group 398 (21.91\%) out of total 1816 drug prescribed to 611 patients (Figure 2). 
- Total $287(46.97 \%)$ patients expired and 307 $(50.24 \%)$ shifted to ward.

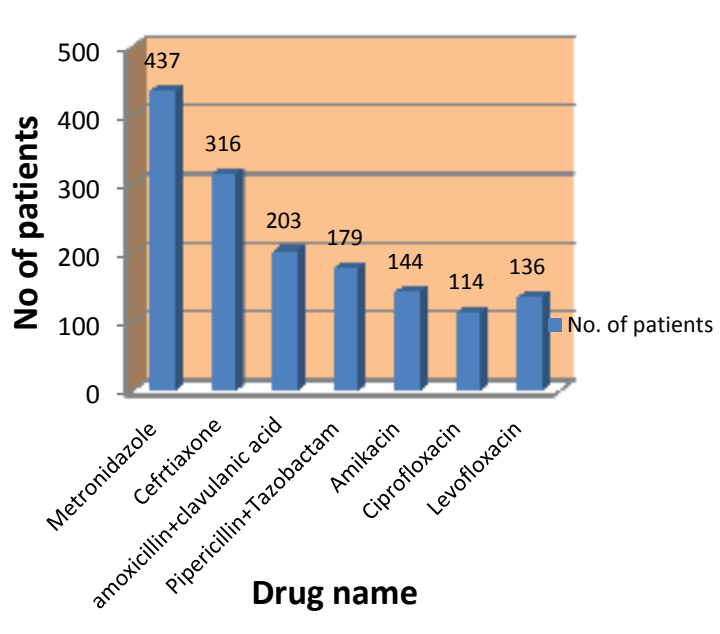

Figure 1: commonly used antimicrobial drugs in ICU.

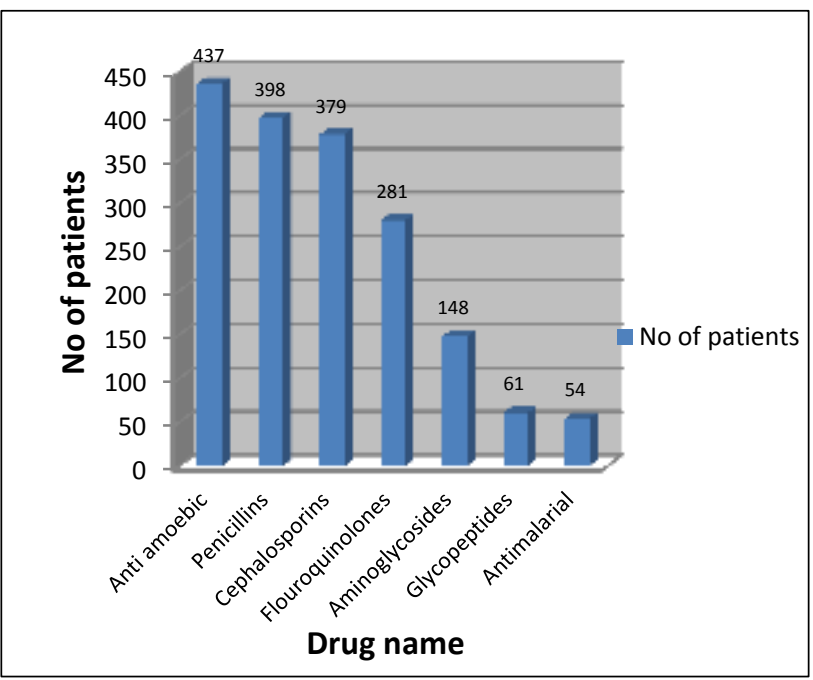

Figure 2: Most common prescribed antimicrobial drug groups.

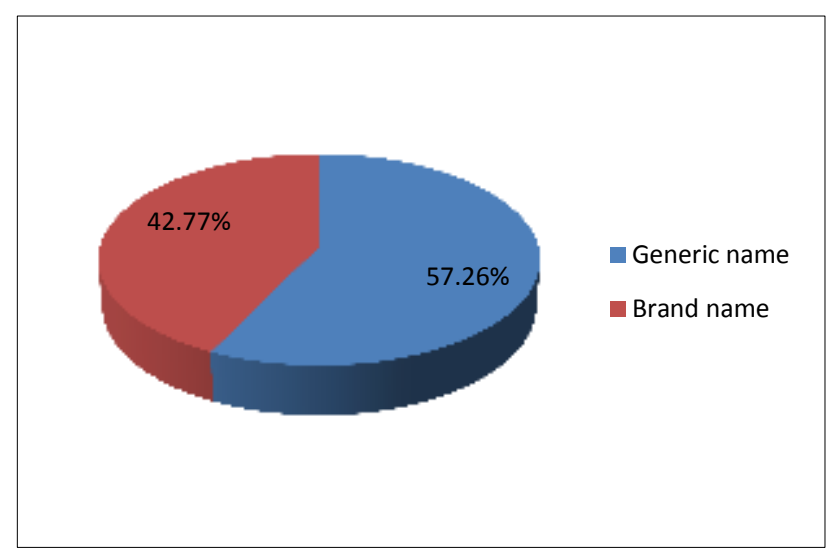

Figure 3: Percentage of antimicrobial drugs prescribed by generic name and brand name.

\section{DISCUSSION}

In our study sample size was 611 .

In our study male to female ratio was $1.51: 1$, male were about $373(61.04 \%)$ and female were of $238(38.95 \%)$ which is similar to male $70(63.63 \%)$ and female $40(36.36 \%)$ other studies. ${ }^{10,11}$

Majority of the patients admitted in ICU were due to post-operative complications $204 \quad$ (33.38\%), oragnophosporhus poisoning $164(26.84 \%)$, accidental trauma $44(7.20 \%)$. While other studies were having most common indication for admission was sepsis. ${ }^{10,12}$

We observed that $287(46.97 \%)$ patients were expired, $307(50.24 \%)$ patients were shifted to ward. While in another studies were having result of $121(60.50 \%)$ discharged, 79 (39.50\%) expired and 204 (28\%) expired, $526(70.6 \%)$ improved respectively. ${ }^{12,13}$

It was observed that average number of antimicrobial drugs prescribed per patient was $2.97 \pm 1.40$ in ICU which was similar to studies where average no. of prescribed antimicrobial drug was 3.36 and 2.09 respectively. ${ }^{14,15}$

It was observed that in our study $1040 \quad(57.26 \%)$ antimicrobial agents were prescribed generically and 776 $(42.70 \%)$ antimicrobial agents prescribed by brand name in ICU. In contrast to other study, showed that $70 \%$ antimicrobial agents were prescribed by brand name. ${ }^{15}$ In other study showed $86 \%$ of drugs were prescribed by brand name. ${ }^{17}$ Our hospital is being tertiary care teaching hospital and medications supplied from central medical store organization, Government of Gujarat. In case of resistance to antimicrobial agent or in case of complication(s) when antimicrobial agents which are not available at hospital, were prescribed from other sources.

Most common group prescribed was antiamoebic drugs $437(24.05 \%)$ in our study followed by penicillin 398 $(21.81 \%)$ and cephalosporin group 379 (20.87\%) which was contrary to other study which shows maximum usage of cephalosporins $409(27 \%)$ followed penicillin group $15.3 \%$ and antiamoebic group $12.6 \% .^{18}$

Most common antimicrobial drug prescribed was metronidazole 437 (24.04\%) followed by ceftriaxone 316 $(17.23 \%)$ and amoxiclav $203(11.16 \%)$ while another study also shows the AMA with maximum consumption was metronidazole $(14.3 \%)$, followed by ceftriaxone $(12.6 \%)$ and artesunate $(10.8 \%){ }^{18}$

In our study average cost per patient for the antimicrobial drug usage is Rs 1805.34. In other study at Uttarakhand revealed average cost per patient for antimicrobial drug is Rs $1995 .^{18}$ While the same $1995.05 \mathrm{Rs} /$ patient for antimicrobial drug usage is seen in other study. ${ }^{13}$ 
Out of 25 drugs $17(68 \%)$ drugs were prescribed according to essential medicine list of WHO, 2013, and National Essential Medicine List (EML) of India (2011). WHO reported that average $60 \%$ drugs prescribed from EML on survey of 35 countries in the world which was lower compare to our study. ${ }^{19}$

\section{CONCLUSION}

Overall, the drugs were prescribed rationally. It gives important conclusion about how antimicrobial drugs are used in GGGH ICU. This information can be used for improvement of current treatment strategies.

\section{ACKNOWLEDGEMENTS}

The authors acknowledge the encouragement, support and valuable suggestions provided by Dr. H. R. Trivedi for preparing the manuscript.

Funding: No funding sources Conflict of interest: None declared

Ethical approval: The study was approved by the Institutional Ethics Committee

\section{REFERENCES}

1. Lee D, Bergmen U. Studies of utilization. In: Strom BC, editor. Pharmacoepidemiology. $1^{\text {st }}$ ed. New York: Churchill Livingstone. 1989:259-73.

2. Introduction to drug utilization research by World Health Organization. http://www.whocc.no/file archive/publications/drug_utilization_research.pdf. Accessed on 2014 Sep10.

3. Gaye U, Schrwartz W. Antimicrobial stewardship programme on hospital acquired infections. Am $\mathbf{J}$ Med. 2005;113:34-9.

4. Marin H, Kunches LM, Lichtenberg DA. Comparison of infections in different ICUs within the same hospital. Crit Care Med. 2005;43(2):122-7.

5. Asem A, Barchitta M Cipresso R, Giaquinta L. Romeo M, Denaro C. Pseudomonas aeruginosa carriage, colonization, and infection in ICU patients. Intensive Care Med. 1996;33:1155-61.

6. Aysen B, Phillips L, Monnet D. The prevalence of nosocomial infection in intensive care units in Europe: results of the European prevalence of infection in intensive care (EPIC) study. JAMA. 2006;274:639-64.

7. Kanhai KK. Non-invasive respiratory monitoring in surgical intensive care. 2001;3:112-34.
8. Gaash B. Irrational use of antimicrobials. Intensive Care Med. 2008;13:342-6.

9. Daniel P, Nyquist AC, Gonzales R, Steiner JF, Sande MA. Antibiotic prescribing for children with colds, upper respiratory tract infections, and bronchitis. JAMA. 2002;279:875-7.

10. Naqvi M, Chiranjeevi U, Shobha J. Prescription patterns of antibiotics in acute medical care unit of a tertiary care hospital in India. Int J Curr Microbiol App Sci. 2014;3:673-9.

11. Vandana A, Sanjaykumar B. Study of prescribing pattern of antimicrobial agents in medicine intensive care unit of a teaching hospital in central India. JAPI. 2012;60:20-3.

12. Lisha J, Padmini D, Jenny J, Shoba G. Drug utilization study of antimicrobial agents in medical intensive care unit of a tertiary care hospital. Asian journal of pharmaceutical and clinical research. 2011;4:81-4.

13. Williams A, Mathai AS, Phillips AS. Antibiotic prescription patterns at admission into tertiary level intensive care unit in Northern India. J pharm Bioall Sci. 2011;3:531-6.

14. Mahajan B, Kaushal S, Chopra S. A drug utilization study of antimicrobial agents (AMAs) in the intensive care units (ICUs) at medical college hospital of North India. J K sci. 2013;15:129-32.

15. Gangwar A, Kumar N, Kothiyal P. Antibiotic prescription and cost patterns in an intensive care unit. The pharma innovation. 2012;1:68-72.

16. John LJ, Devi P John P, Guido S. Drug utilization study of antimicrobial agents in medical intensive care unit. Asian J Pharm Clin Res. 2011;4(2):81-4.

17. Saleh NA, Purya M, Hemadri M. Generic names or trade names prescribing practices of junior doctors. Kuwait Med J. 2001;33(2):153-5.

18. Bansal D, Mangla S, Undela K, Gudala K, D'Cruz S, Sachdev A, Tiwari P. Measurement of adult antimicrobial drug use in tertiary care hospital using defined daily dose and days of therapy. Indian $\mathbf{J}$ Pharm Sci. 2014;76:211-7.

19. WHO. The world medicine situation. Geneva: WHO press, World Health Organization; 2004:1-25 http://apps.who.int/medicinedocs/pdf/s6160e/s6160e .pdf. Accessed on 30 ${ }^{\text {th }}$ March 2015.

Cite this article as: Hedamba R, Doshi C, Darji NH, Patel B, Kumari V, Trivedi HR. Drug utilization pattern of antimicrobial drugs in intensive care unit of a tertiary care hospital attached with a medical college. Int J Basic Clin Pharmacol 2016;5:169-72. 